\title{
Burden of pneumocystis pneumonia in HIV-infected adults in sub-Saharan Africa: a systematic review and meta-analysis
}

\author{
Sean Wasserman ${ }^{*}$, Mark E. Engel ${ }^{2}$, Rulan Griesel ${ }^{3}$ and Marc Mendelson ${ }^{1}$
}

\begin{abstract}
Background: Seroprevalence data and clinical studies in children suggest that the burden of pneumocystis pneumonia (PCP) in Africa may be underestimated. We performed a systematic review to determine the prevalence and attributable mortality of PCP amongst HIV-infected adults in sub-Saharan Africa.

Methods: We searched Pubmed, Web of Science, Africa-Wide: NiPAD and CINAHL, from Jan 11995 to June 1 2015, for studies that reported the prevalence, mortality or case fatality of PCP in HIV-infected adults living in sub-Saharan African countries. Prevalence data from individual studies were combined by random-effects meta-analysis according to the Mantel-Haenszel method. Data were stratified by clinical setting, diagnostic method, and study year.

Results: We included 48 unique study populations comprising 6884 individuals from 18 countries in sub-Saharan Africa. The pooled prevalence of PCP among 6018 patients from all clinical settings was $15 \cdot 4 \%(95 \% \mathrm{Cl} 12 \cdot 9-18 \cdot 0)$, and was highest amongst inpatients, $22 \cdot 4 \%(95 \% \mathrm{Cl} 17 \cdot 2-27 \cdot 7)$. More cases were identified by bronchoalveolar lavage, $21 \cdot 0 \%(15 \cdot 0-27 \cdot 0)$, compared with expectorated, $7 \cdot 7 \%(4 \cdot 4-11 \cdot 1)$, or induced sputum, $11 \cdot 7 \%(4 \cdot 9-18 \cdot 4)$. Polymerase chain reaction (PCR) was used in 14 studies $(n=1686)$. There was a trend of decreasing PCP prevalence amongst inpatients over time, from $28 \%(21-34)$ in the 1990 s to $9 \%(8-10)$ after 2005 . The case fatality rate was $18 \cdot 8 \%(11 \cdot 0-26 \cdot 5)$, and PCP accounted for $6 \cdot 5 \%(3 \cdot 7-9 \cdot 3)$ of study deaths.

Conclusions: PCP is an important opportunistic infection amongst HIV-infected adults in sub-Saharan Africa, particularly amongst patients admitted to hospital. Although prevalence appears to be decreasing, improved access to antiretroviral therapy and non-invasive diagnostics, such as PCR, are needed.
\end{abstract}

Keywords: Pneumocystis pneumonia, HIV-associated opportunistic infection, Respiratory disease in HIV, PCP

\section{Background}

Pneumocystis jirovecii is a ubiquitous opportunistic fungal pathogen that causes pneumocystis pneumonia (PCP) in patients with cellular immune defects. PCP heralded the onset of the global human immunodeficiency virus (HIV) pandemic [1], and prior to 1995 it was estimated that two-thirds of HIV-infected persons would eventually develop PCP [2]. Although the incidence has declined since the introduction of cotrimoxazole prophylaxis and combination antiretroviral therapy (ART), PCP remains the most important AIDS-defining opportunistic infection

\footnotetext{
* Correspondence: sean.wasserman@uct.ac.za

${ }^{1}$ Division of Infectious Diseases and HIV Medicine, Department of Medicine, University of Cape Town, Cape Town, South Africa

Full list of author information is available at the end of the article
}

in the United States [3, 4]. By contrast, in sub-Saharan Africa the morbidity and mortality in HIV is dominated by tuberculosis [5], cryptococcal disease, and bacterial infections such as non-typhoidal salmonella and Streptococcus pneumoniae [6].

Early studies from African countries with high HIV prevalence reported PCP to be an uncommon OI [7-9]. An overwhelmingly high burden of other opportunistic diseases, early exposure to $P$. jirovecii resulting in improved immunity, and immune modulation by other infections are putative explanations for the reduced prevalence of PCP in sub-Saharan Africa [10-12]. A single recent study demonstrated an inverse relationship of PCP prevalence to gross domestic product [13]. 
In the context of late presentation at low CD4 T lymphocyte counts (CD4 count) [14] and limited ART coverage [15], there is potentially a large pool of individuals at high risk of presenting to health care facilities with PCP in sub-Saharan Africa. Local studies have revealed a high seroprevalence of $P$. jirovecii in healthy HIV-infected adults $[16,17]$, as well as high rates of clinical disease in African children [18-20]. The reported increase in prevalence amongst adults in the 1990s, possibly as a result of better access to diagnostics and treatment [21], may reflect the true epidemiology of the disease, suggesting that PCP may be a more important cause of community-acquired pneumonia in Africa than was previously recognised.

A better understanding of PCP prevalence will help to inform empiric prescribing, the interpretation of diagnostic tests, and public health policy relating to the allocation of scarce resources such as intensive care unit beds, invasive procedures like bronchoscopy, and research funding. We therefore undertook a systematic review to more accurately determine the burden of PCP in sub-Saharan Africa in the ART era. Our primary aim was to determine the proportion of HIV-infected adults with confirmed PCP, stratified by clinical setting, method of diagnosis, and time. Secondary aims included attributable mortality and case fatality, a description of demographic profiles, and rates of co-infection with other pathogens.

\section{Methods}

\section{Search strategy and selection criteria}

A detailed protocol describing our methods has been published elsewhere [22] and is registered in the Prospero database (CRD42013005530). We developed a broad search strategy to identify studies that reported the prevalence, mortality or case fatality of PCP in sub-Saharan Africa. Our systematic review used the search terms "pneumocystis," "P jirovecii," "P carinii," "pneumonia," "HIV," "Africa south of the Sahara," and related terms for relevant studies published in Pubmed, Web of Science, Africa-Wide: NiPAD and CINAHL between 1995 and 1 June 2015 (the full search strategy is included in the supplementary appendix, Additional file 1). No language or age limits were applied. We hand-searched bibliographies of all recovered articles for potentially eligible studies and contacted authors for unpublished data. The year 1995 was chosen as a cut-off because previous reviews have reported on PCP prevalence from early studies in sub-Saharan Africa, and we wanted to assess the impact of more widespread availability of cotrimoxazole prophylaxis and ART. Titles and abstracts of references recovered by the search were screened and the full texts of potentially relevant articles were independently assessed by two reviewers (SW and RG) using a standardised score sheet. Disagreements on final inclusions were resolved by discussion and consensus involving a third reviewer (MM).
Studies assessing a clearly defined population of HIVinfected adults ( $\geq 13$ years of age, chosen because the diagnosis and treatment of PCP in this age group is identical to older adults, and 13 years is the age of transition into adult care in the public health sector in South Africa) in any clinical setting were included if they applied specific diagnostic criteria for PCP. These were predefined with the use of a novel quality-scoring tool, and included either a clear clinical case definition (based on World Health Organization criteria [23]) or confirmation with laboratory testing (using histochemical or fluorescent staining or molecular assays). Reports involving cohorts of patients with PCP were ineligible for primary analysis of pooled prevalence, but were included if they provided data on case fatality.

\section{Data extraction}

Data were independently extracted by two reviewers (SW and RG) and compared for consistency using pre-specified scores derived for each main eligibility category. The key variable was the proportion of HIV-infected adults in the study cohort diagnosed with PCP. As a minority of studies were specifically designed to assess this outcome, our denominator was defined as the population of patients who underwent clinical or laboratory evaluation for PCP. Prevalence was defined as the number of cases of PCP diagnosed among patients with signs and symptoms of PCP who would normally warrant testing at a given hospital or clinic, and not the population prevalence of disease. We defined mortality and case fatality as the proportion of deaths due to PCP (PCP deaths/total deaths) and mortality amongst PCP cases (PCP deaths/total PCP diagnoses), respectively.

Data were stratified by clinical setting. As an indicator of severity and as a crude estimate of the pre-test probability of PCP, we categorised in- and outpatients into separate groups, given that susceptible inpatients with respiratory symptoms are more likely to have a diagnosis of PCP and to have more severe disease than outpatients, which will influence PCP prevalence and mortality. Studies that recruited both in- and outpatients, but from which the respective proportions of these groups could not be ascertained, were classified separately. We also stratified data by diagnostic method and clinical specimen.

Patients with symptoms compatible with PCP plus any positive diagnostic test or fulfilling criteria for the clinical case definition were considered to have confirmed disease, and were included in our primary analysis. Asymptomatic individuals or those with an illness that resolved without specific PCP therapy, but had a positive $P$. jirovecii PCR (any assay) were defined as being colonised.

Where available, the following information was captured; CD4 count, numbers of patients on cotrimoxazole 
prophylaxis and/or ART, type and frequency of co-infection(s), relevant data from studies with duplicate cohorts that were excluded from the pooled prevalence analysis, and demographic details.

\section{Methodological quality of included studies}

Overall quality of included studies was evaluated using our published scoring system [22]. The tool assigns numerical scores to the following domains: participant selection (study design and objectives), outcome ascertainment (diagnostic criteria) and quality of data available for extraction. We used the tool to code and standardise study eligibility decisions and to assess agreement between investigators. As this is a prevalence review, we assessed studies for selection bias and overall bias, using criteria defined by the Cochrane group (Review Manager Version 5.2, http:// ims.cochrane.org/RevMan).

\section{Data analysis}

Point estimates and $95 \%$ confidence intervals were derived for all outcomes using prevalence data from included studies. Meta-analysis was performed according to the Mantel-Haenszel method applying the random-effects model to account for between study variability. We evaluated heterogeneity using the $\chi 2$-based $\mathrm{Q}$ statistic (significant for $p<0 \cdot 1)$ and the $\mathrm{I}^{2}$ statistic ( $>50 \%$ indicative of "notable" heterogeneity) [24]. STATA software version 11 (STATA Corporation, College Station, Texas) was used to perform calculations and the meta-analysis and to produce the forest plots using the metan routine. Where standard errors (SE) were not provided, we incorporated confidence intervals into the formula, $\mathrm{SE}=$ (upper limit - lower limit) $/$ $3 \cdot 92$.

\section{Results}

\section{Study characteristics}

Our search identified 1862 records, with an additional five studies added from searching bibliographies. After removing duplicates and screening abstracts for relevance to the study aims, we evaluated 185 full text articles for eligibility, ultimately including 48 unique study populations (Fig. 1; a full reference list of included studies available in
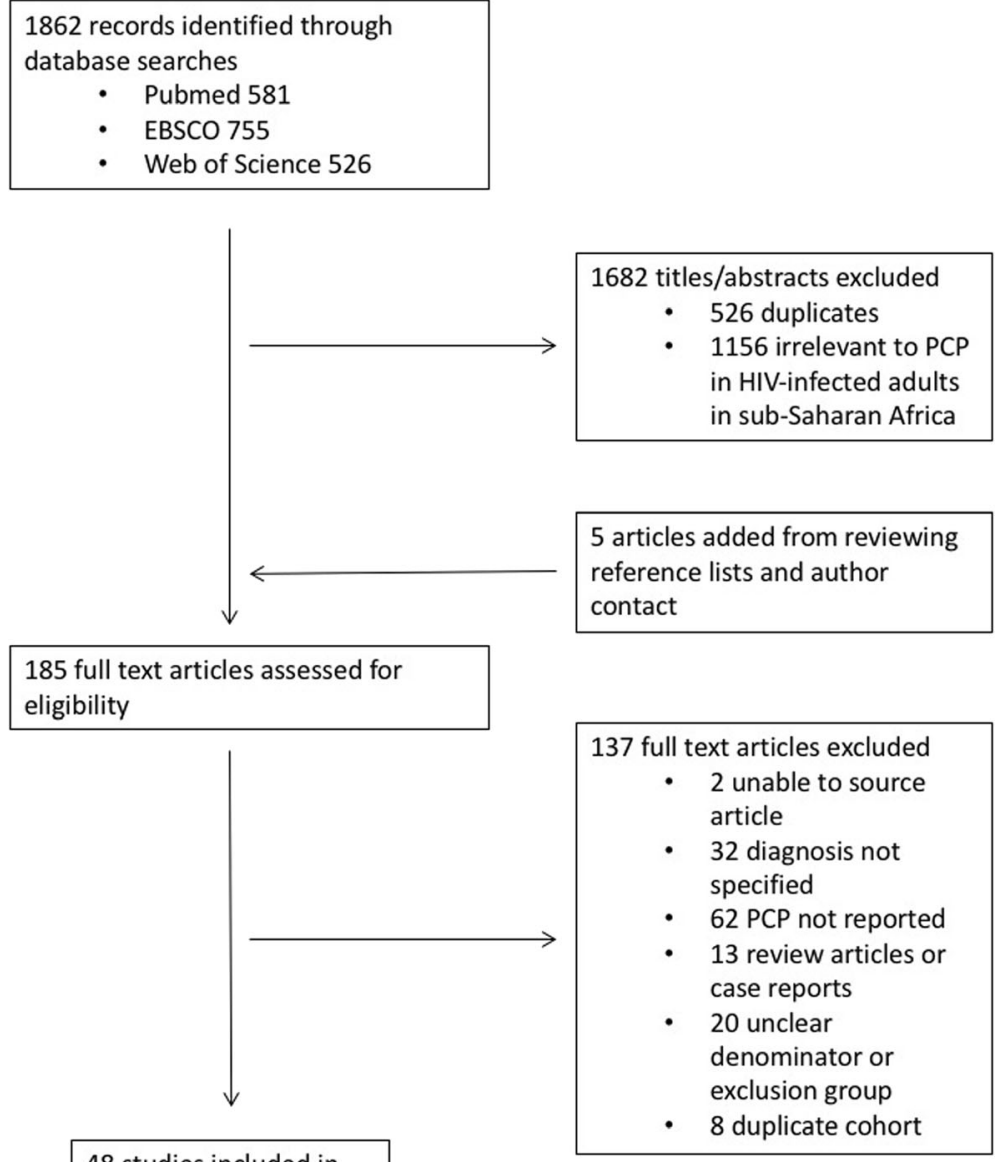

48 studies included in the review

Fig. 1 PRISMA flow diagram 
the online supplementary appendix, Additional file 2). Of these, 35 studies (6018 patients) reported the prevalence of PCP from clinical cohorts; eleven were post-mortem studies (707 deaths) and two provided data on clinical characteristics and case fatality only. For the studies with a clear denominator to evaluate PCP prevalence, the pre-defined clinical categories were represented as follows: inpatients, 23 studies $(n=2593)$; outpatients, 7 studies $(n=2656)$; and enrolment of both in- and outpatients, 5 studies $(n=769)$. The dataset included patients from 18 sub-Saharan African countries: Benin, Burkina Faso, Central African Republic, Mali, Mozambiqe, Nigeria, Namibia and Zambia (1 study); Botswana and Zimbabwe (2 studies); Côte d' Iviore, Ethiopia, Malawi, Senegal and Tanzania (3 studies); Kenya (4 studies); Uganda (8 studies); South Africa (13 studies). Apart from 3 clinical studies that recruited patients from district hospitals, all others were conducted in tertiary or central hospital referral centres.

Study characteristics are summarised in the online supplementary appendix (Additional file 3). The median CD4 count of patients assessed for PCP was 75 cells/ $\mathrm{mm}^{3}$ (interquartile range (IQR) 63-145; 20 studies, $n=$ 3543). $18 \cdot 7 \%$ (983/5249) were on ART (26 studies, $n=$ $5249)$ and $34.4 \%(1377 / 4002)$ were receiving PCP prophylaxis (21 studies, $n=4002)$.

Although most studies $(42, n=6285)$ were prospective in design the overall data quality was poor: risk of bias was assessed as high in 30 studies $(n=4346)$ and 19 studies $(n=4140)$ were assigned an overall quality assessment score of $\leq 9$ (poor quality) (available in the online supplementary appendix, Additional file 4). Agreement between investigators with regard to decisions about applied qualitative scores was acceptable (interrater agreement = $95 \cdot 12 \%$, kappa $=0 \cdot 8292$ ).

\section{PCP prevalence}

The overall prevalence of PCP among 6018 patients in 35 clinical studies was 15.4\% (95\% confidence interval (CI), 12.9-18.0) (Fig. 2). Heterogeneity was high $(p<0 \cdot 1$; $\left.\mathrm{I}^{2}=95 \cdot 6 \%\right)$. Among all patients presenting with respiratory symptoms the prevalence was $18.8 \%$ (31 studies, $n=3504,95 \% \mathrm{CI}, 14 \cdot 9-22 \cdot 6)$, and $17 \cdot 1 \%$ (21 studies, $n=2086,95 \%$ CI 12.5-21.7) in studies that evaluated patients who were sputum smear-negative for acid-fast bacilli.

The proportions of PCP cases stratified by clinical setting, post mortem reports, time period studied and diagnostic methods used, are shown in Fig. 3. By setting, prevalence was highest amongst inpatients $(22 \cdot 4 \%, 23$ studies, $n=2593,95 \%$ CI 17.2-27.7) compared with outpatients $(4 \cdot 8 \%, 7$ studies, $n=2656,95 \%$ CI 2 $7-6 \cdot 9)$ and patients recruited from both in- and outpatient settings $(11 \cdot 5 \%, n=769,95 \%$ CI $4 \cdot 1-19 \cdot 0)$. PCP was diagnosed in $24 \%$ (22 studies, $n=1769,95 \%$ CI $17 \cdot 8$ $30 \cdot 3$ ) of inpatients with respiratory symptoms.

Figure 3a shows the comparison of PCP prevalence for inpatients by the year that data was last collected in the study. The pooled percentages of PCP cases amongst inpatients over three time periods were as follows: 1990s, 28 \% (4 studies, $n=192,95 \%$ CI 21-34), 2000-2004, $27 \%$ (8 studies, $n=633,95 \%$ CI 23-30), 2005 and later, $9 \%$ (11 studies, $n=1768,95 \%$ CI 8-10).

The proportion of inpatients on either ART or cotrimoxazole in the two earlier time periods was $3.6 \%$ $(n=276,95 \%$ CI $1 \cdot 8-6 \cdot 6)$ and $2.6 \%(n=227,95 \% \mathrm{CI}$ $0 \cdot 98-5 \cdot 7)$ respectively, and the proportions in the years after 2005 were $24.3 \%(n=1482,95 \%$ CI $9 \cdot 8-38 \cdot 7)$ and $30 \cdot 4 \%(n=1421,95 \% \mathrm{CI} 11 \cdot 6-49 \cdot 3)$ respectively.

PCP prevalence according to diagnostic method is shown in Fig. 3b. The prevalence for clinical specimens diagnosed by BAL was $21 \cdot 0 \%(n=1591,95 \%$ CI $15 \cdot 0-27 \cdot 0)$; this was significantly higher than from expectorated sputum $(7 \cdot 7 \%, n=740,95 \%$ CI $4 \cdot 4-11 \cdot 1)$, and numerically higher than from induced sputum $(11.7 \%, n=553,95 \%$ CI 4 9-18 -4). The type of clinical specimen was not specified in 3 studies ( $n=445$, PCP cases 40$)$.

\section{Case fatality, mortality and clinical characteristics}

The clinical characteristics of PCP cases, proportions and type of co-infections are listed in table 1 . The case fatality was $18 \cdot 8 \%(95 \%$ CI $11 \cdot 0-26 \cdot 5$; range $0-71$. $4 \%$ ); data for this calculation were available from 12 studies $(n=334)$, representing fewer than half $(334 / 716)$ of all confirmed cases. Only two studies $(n=16)$ reported follow up times; 11 patients were followed for 1 month $(0$ deaths) and five followed for 2 months post-discharge (case fatality $60 \%$ ). All other PCP-related deaths occurred during the index admission. $29.5 \%$ of the 474 PCP cases in whom additional testing was performed had a confirmed co-existent opportunistic pulmonary disease. Tuberculosis was most common (14.8\%), followed by bacterial pneumonia (8.7\%) and Kaposi sarcoma (4.1\%).

Where data were available, PCP accounted for $6.5 \%$ (95\% CI 3.7-9.3) of the overall mortality (17 studies, $n=1110$ ). Post-mortem studies provided data for 707 deaths of which $5.9 \%(95 \%$ CI 3.2-8.6) were attributed to PCP. Apart from one study that evaluated all-cause maternal mortality, the primary objectives of other post-mortem studies included the determination of cause of death in HIV-infected adults. Three of these studies $(n=221)$ enrolled subjects with pre-morbid respiratory symptoms, in whom PCP-related mortality was $10 \%$ (95 \% CI 6-14).

\section{Discussion}

Early studies conducted in sub-Saharan Africa suggested a lower PCP prevalence than in developed countries 


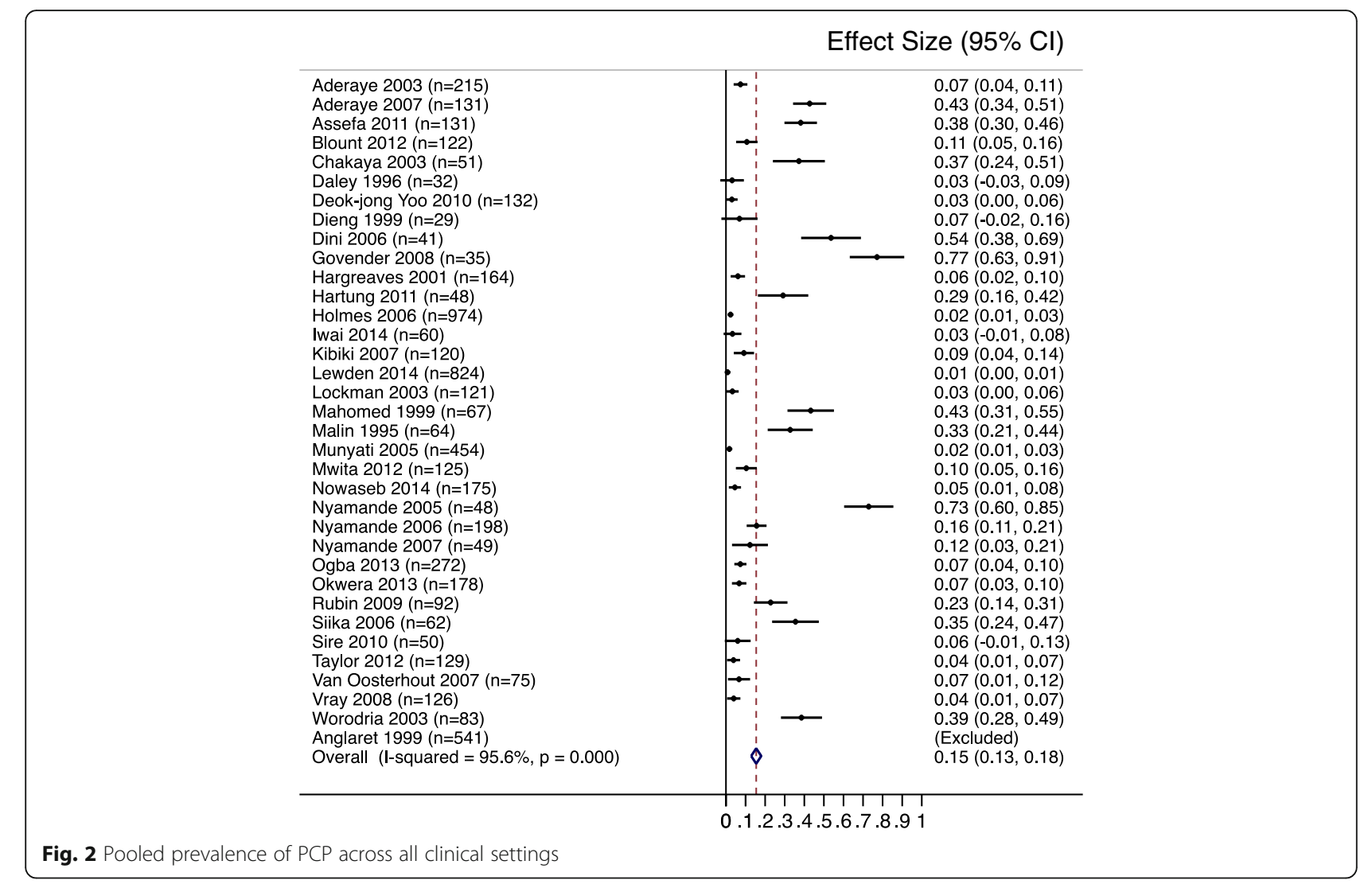

[25-27], as well as a lower incidence (5 cases per 1000 person years) compared to the post-ART era estimate in the United States of 46 cases per 1000 person years [28]. These data have led a number of experts to question the importance of PCP in comparison to tuberculosis and other infections that dominate mortality and morbidity in sub-Saharan Africa [10-12]. Our review of data collected predominantly after 1995 demonstrates a pooled prevalence of PCP comparable to that reported from developed countries [29], suggesting that it is more likely to have been missed or neglected in sub-Saharan Africa rather than being an uncommon opportunistic infection.

Previous reviews have shown time trend of increasing PCP prevalence in sub-Saharan Africa amongst comparable patient populations and diagnostic assessments. After crudely combining the data from these reports, seven out of ten studies conducted over the period 1986-1993 had prevalence rates $\leq 15 \%$ while eight out of ten studies conducted between 1997 and 2005 had prevalence rates $\geq 30 \%[21,30]$. We have shown the opposite, that the proportion of inpatients with PCP appears to be decreasing over time. This trend seems more realistic, and it is plausible that our observation of better exposure to ART and cotrimoxazole prophylaxis in more recent years can account for fewer diagnosed cases of PCP. Nonetheless, even after 2005, most patients in our included studies had not received these interventions and had low CD4 counts at presentation, reflecting the slow rollout of adequate HIV care in the region.

We noted a much higher prevalence of PCP amongst inpatients compared to outpatients. This is mainly a reflection of the superior performance of invasive diagnostic tests and that inpatients represent a selected denominator of patients that have failed empiric therapy for other causes of pneumonia in the community. However, the observation may also suggest something inherent about PCP itself, possibly that mild cases either resolve spontaneously or are an uncommon manifestation of the infection; these hypotheses require further study.

More cases of PCP were diagnosed using BAL (21\%) compared with sputum (7-11\%). This reflects a selection bias of patients with a higher likelihood of PCP being referred for bronchoscopy but also the enhanced sensitivity of BAL for diagnosis. Unfortunately, access to bronchoscopy is extremely limited in most African countries, and clinicians must rely on a combination of knowledge of disease prevalence, unvalidated clinical case definitions and insensitive non-invasive tests to 


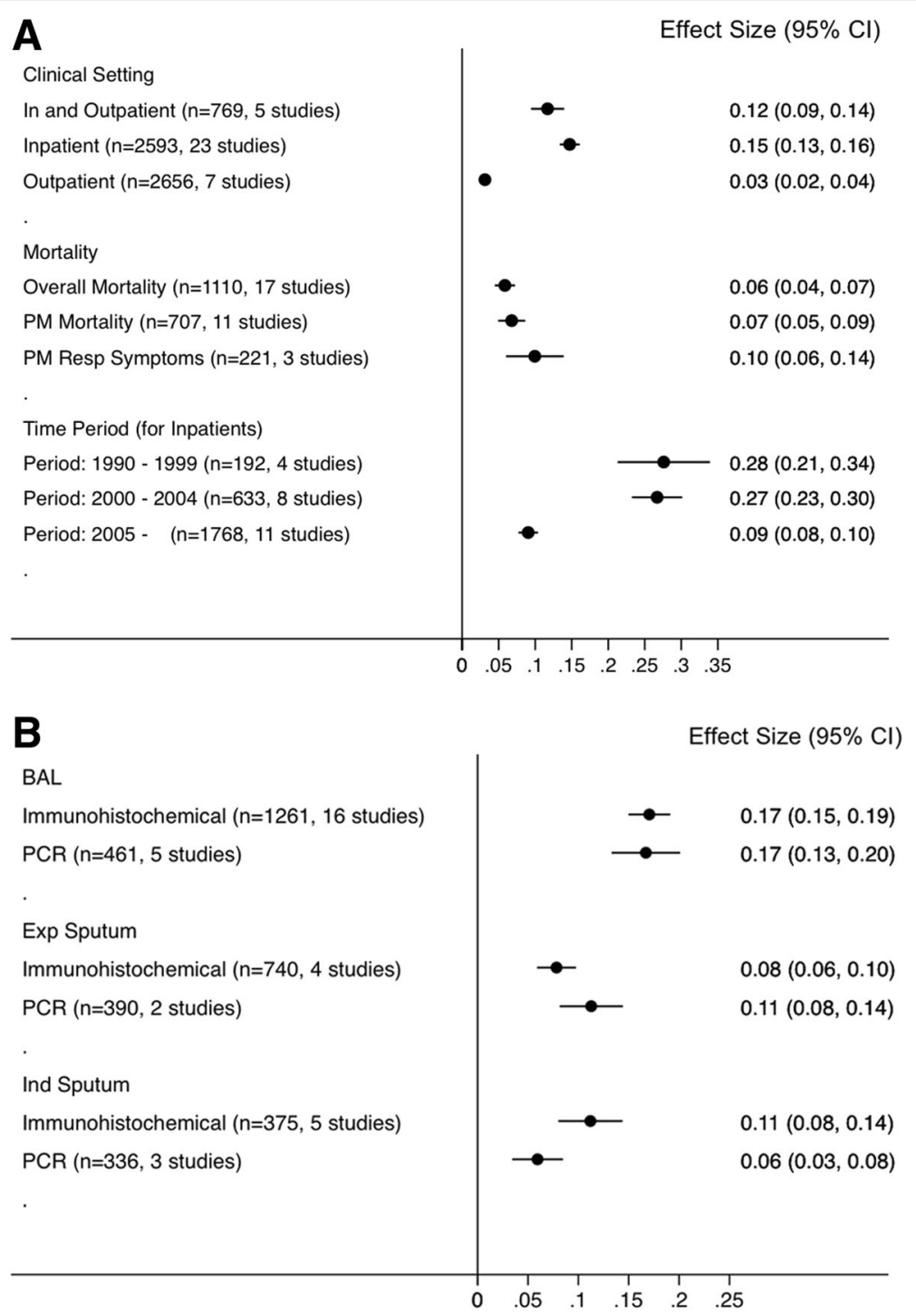

Fig. 3 Forest plots of proportion of patients with PCP by clinical setting, mortality and time period (a) and by diagnostic method (b). PM post mortem, resp respiratory $B A L$ bronchoalveolar lavage, $P C R$ polymerase chain reaction, Exp expectorated, Ind induced. Heterogeneity: A: Clinical Setting, $P<$ $0.1 ; I^{2}=99.4 \% ;$ Mortality, $p=0.83 ; I^{2}=0.0 \%$; Time Period, $p<0.1 ; I^{2}=91.8 \%$ B: BAL, $p=0.62 ; I^{2}=0.0 \%$; expectorated sputum, $p=0.51 ; I^{2}=0.0 \%$; induced sputum, $p<0.1 ; 1^{2}=71.9 \%$

diagnose PCP. In the context of a prevalence of $>20 \%$ and poor diagnostic tools, there should be a low threshold to initiate empiric PCP therapy for HIV-infected inpatients with pneumonia. This may lead to overtreatment, with the attendant risks of unnecessary exposure to high dose cotrimoxazole and concerns about using corticosteroids in patients with undiagnosed tuberculosis or Kaposi sarcoma. Our finding of a high frequency of PCP with the use of bronchoscopy therefore emphasises the need for more accurate non-invasive diagnostic tests such as PCR, which has been shown to be sensitive, specific and cost effective when used on sputum and BAL [31], with a potentially important role as a rule-out test for PCP in resource-limited settings [32]. Only ten studies included in our review used PCR assays to diagnose PCP, and similar numbers of cases were identified by this method and staining techniques on all specimen types. Our study was not designed to assess the performance of diagnostic tests for $\mathrm{PCP}$, and prospective cohort studies are needed to evaluate the role of PCR, and other noninvasive tests such as plasma beta-glucan [33], in the diagnosis of PCP in sub-Saharan Africa.

Our calculated case fatality of almost $19 \%$ is higher than the mortality from other common respiratory infections in Africa [34, 35] as well as from PCP in developed countries [36]. This contributes to the overall burden of PCP and adds impetus to more investment in prevention strategies and effective diagnostic tests. 
Table 1 Characteristics of all PCP cases $(n=743)$

\begin{tabular}{|c|c|}
\hline Age (years, mean) & 35 (10 studies, $n=240$ ) \\
\hline Male sex (\%) & $39.6(15$ studies, $n=315)$ \\
\hline $\begin{array}{l}\text { CD4 count (cells/microL, } \\
\text { median, IQR) }\end{array}$ & $48[31.5-129.0]$ (16 studies, $n=353)$ \\
\hline $\begin{array}{l}\text { Cotrimoxazole prophylaxis } \\
(\%, 95 \% \mathrm{Cl})\end{array}$ & 5.9 [3.3-9.5] (11 studies, $n=255)$ \\
\hline ART $(\%, 95 \%$ Cl) & 0.4 [0.01-2.6] (11 studies, $n=215)$ \\
\hline \multicolumn{2}{|c|}{ Co-existent opportunistic disease (\%, $95 \%$ Cl) } \\
\hline - Overall & $29.3[25.4-33.6](26$ studies, $n=474)$ \\
\hline - Tuberculosis & $14.8[11.8-18.5](25$ studies, $n=431)$ \\
\hline - Bacterial pneumonia & 8.7 [0.6-11.8] (22 studies, $n=445)$ \\
\hline - Pulmonary cryptococcosis & $1.4[0.4-3.6]$ (17 studies, $n=283$ ) \\
\hline - Pulmonary Kaposi sarcoma & 4.1 [2.4-6.6] (21 studies, $n=410)$ \\
\hline - Pulmonary CMV & 3.9 [1.4-8.2] (11 studies, $n=155)$ \\
\hline - Pulmonary MAC & $0.8[0.1-2.8]$ (19 studies, $n=254)$ \\
\hline Case fatality rate $(\%, 95 \% \mathrm{Cl})$ & $18.8 \%[11.0-26.5]$ (12 studies, $n=334)$ \\
\hline
\end{tabular}

IQR interquartile range; $C l$ confidence interval; $A R T$ antiretroviral therapy; $C M V$ cytomegalovirus; $M A C$ mycobacterium avium complex

Similarly to a recent review [13], a substantial number of patients with PCP were found to have coexistent bacterial pneumonia and other opportunistic infections, particularly tuberculosis. This is an important consideration when evaluating patients with inadequate responses to empiric or confirmed PCP therapy, and co-infections should be excluded before attributing a poor treatment response to $\mathrm{PCP}$ infection alone.

This review has a number of important limitations. First, most included studies were conducted in highly selected cohorts, such as patients with suspected PCP, exclusion of patients with tuberculosis and other illnesses, and the performance of BAL only in cases where the diagnosis remained elusive. This increases the risk of selection bias and of overestimating true disease prevalence. However, the clinical burden of PCP in a population is influenced almost exclusively by those who are ill with respiratory symptoms and our chosen denominator therefore allows an assessment that is probably close to the true prevalence.

Second, missing or unreported data was common. With regard to disease prevalence, bronchoscopy studies frequently excluded a substantial proportion of patients with possible PCP on the basis of medical contraindications for the procedure, and five studies excluded patients with either advanced HIV or severe respiratory illness. If anything, this may result in an underestimate of PCP prevalence because the outcomes of otherwise eligible patients with respiratory symptoms not enrolled were rarely reported and over half of the included studies did not specifically evaluate participants for PCP.

The third limitation was the high degree of heterogeneity in the prevalence findings from different studies. This is not unusual in prevalence reviews, and reflects differences in methodology and aims, diagnostic methods and the chosen population denominator. We attempted to minimise this effect by analysing data by clinical setting to include patients with similar risk for PCP and by scoring the quality of each study, and found no significant difference in prevalence on the basis of study quality (data not shown). It is also possible that some undefined environmental or biological differences exist across geographic locations to explain the observed heterogeneity, but our study was not designed to address this.

\section{Conclusions}

We have performed a systematic review of PCP prevalence using diagnostic criteria with high specificity, a well-defined population denominator and clinically relevant categorisation of cases. The overall impression from our pooled data is that PCP is an important cause of community-acquired pneumonia in HIV-infected adults living in sub-Saharan Africa. This has implications for clinical guidelines in resource poor settings where indications for empiric therapy and referral need to be clearly defined. The contribution of PCP to mortality in the region suggests that cases are unrecognised, and highlights the need for the development of a reliable clinical case definition and the evaluation of sensitive non-invasive diagnostic tests, such as PCR. The observed trend of decreasing PCP prevalence is encouraging, but there is much work to be done to expand access to ART and cotrimoxazole prophylaxis so that ultimately this sentinel AIDS-defining infection achieves the status of a rare disease it was once erroneously ascribed.

\section{Additional files}

Additional file 1: Table S1. Full search strategy. (PDF 817 kb)

Additional file 2: Full reference list of included studies. (DOCX 121 kb)

Additional file 3: Summary table of included study cohorts. (DOCX $158 \mathrm{~kb}$ )

Additional file 4: Figure showing bias and quality assessment. (PPTX $93 \mathrm{~kb}$ )

Acknowledgements

Not applicable.

Funding

No financial support was received for this study.

Availability of data and material

All data analysed during this study are included in this published article (and its supplementary information files), and are available from included studies which are fully referenced. 


\section{Authors' contributions}

SW designed and performed the literature search, extracted and anaylsed the data, and wrote the manuscript. MEE performed the meta-analysis, generated the figures and edited the manuscript. RG helped with the literature search and data extraction. MM conceived the study and edited the manuscript. All authors read and approved the final manuscript.

\section{Competing interests}

The authors declare that they have no competing interests.

\section{Consent for publication}

Not applicable.

\section{Ethics approval and consent to participate}

This was a systematic review of published literature. All included studies obtained relevant ethics approvals, which was therefore not required for this review.

\section{Author details}

'Division of Infectious Diseases and HIV Medicine, Department of Medicine, University of Cape Town, Cape Town, South Africa. ${ }^{2}$ Department of Medicine, University of Cape Town, Cape Town, South Africa. ${ }^{3}$ Division of Clinical Pharmacology, Department of Medicine, University of Cape Town, Cape Town, South Africa.

\section{Received: 10 February 2016 Accepted: 26 August 2016} Published online: 09 September 2016

\section{References}

1. Masur H, Michelis MA, Greene JB, Onorato I, Stouwe RA, Holzman RS, et al. An outbreak of community-acquired Pneumocystis Carinii pneumonia: initial manifestation of cellular immune dysfunction. N Engl J Med. 1981;305(24): 1431-8. doi:10.1056/Nejm198112103052402.

2. Hay JW, Osmond DH, Jacobson MA. Projecting the medical costs of aids and arc in the United States. J Acquir Immune Defic Syndr. 1988; 1(5):466-85

3. Morris A, Lundgren JD, Masur H, Walzer PD, Hanson DL, Frederick T, et al. Current epidemiology of Pneumocystis pneumonia. Emerg Infect Dis. 2004; 10(10):1713-20. doi:10.3201/Eid1010.030985.

4. Kelley CF, Checkley W, Mannino DM, Franco-Paredes C, Del Rio C, Holguin F. Trends in hospitalizations for aids-associated Pneumocystis Jirovecii pneumonia in the United States (1986 to 2005). Chest. 2009;136(1):190-7. doi:10.1378/Chest.08-2859.

5. World Health Organization. Global tuberculosis report 2014. Geneva: World Health Organization; 2014.

6. Holmes CB, Losina E, Walensky RP, Yazdanpanah Y, Freedberg KA. Review of human immunodeficiency virus type 1-related opportunistic infections in Sub-Saharan Africa. Clin Infect Dis. 2003;36(5):652-62. doi:10.1086/367655. Epub 2003/02/21.

7. Serwadda D, Goodgame R, Lucas S, Kocjan G. Absence of Pneumocystosis in Ugandan aids patients. Aids. 1989;3(1):47-8.

8. Elvin KM, Lumbwe CM, Luo NP, Bjorkman A, Kallenius G, Linder E. Pneumocystis Carinii is not a major cause of pneumonia in HIV infected patients in Lusaka, Zambia. Trans R Soc Trop Med Hyg. 1989; 83(4):553-5.

9. Karstaedt AS. Aids-The Baragwanath experience. Part III. HIV infection in adults At Baragwanath Hospital. S Afr Med J. 1992:82(2):95-7.

10. Maartens G. Opportunistic infections associated with HIV infection in Africa. Oral Dis. 2002;8 Suppl 2:76-9. Epub 2002/08/08.

11. Zar HJ, Maartens G, Wood R, Hussey G. Pneumocystis Carinii pneumonia in HIV-infected patients in Africa-an important pathogen? S Afr Medl J = Suid-Afrikaanse Tydskrif Vir Geneeskunde. 2000;90(7):684-8. Epub 2000/09/14

12. Russian DA, Kovacs JA. Pneumocystis Carinii in Africa: an emerging pathogen? Lancet. 1995;346(8985):1242-3. Epub 1995/11/11.

13. Lowe DM, Rangaka MX, Gordon F, James CD, Miller RF. Pneumocystis Jirovecii pneumonia in tropical and low and middle income countries: a systematic review and meta-regression. Plos One. 2013;8(8):E69969. doi:10.1371/Journal. Pone.0069969.
14. Siedner MJ, Ng CK, Bassett IV, Katz IT, Bangsberg DR, Tsai AC. Trends in CD4 count at presentation to care and treatment initiation in Sub-Saharan Africa, 2002-2013: A meta-analysis. Clin Infect Dis. 2014. doi:10.1093/Cid/Ciu1137. Epub 2014/12/18.

15. World Health Organization. Antiretroviral therapy (ART) coverage among all age groups. Available at: http://www.who.int/gho/hiv/epidemic response/ ART_text/en/. Accessed 31 Aug 2016.

16. Nkinin SW, Daly KR, Walzer PD, Ndzi ES, Asonganyi T, Respaldiza N, et al. Evidence for high prevalence of Pneumocystis Jirovecii exposure among Cameroonians. Acta Trop. 2009;112(2):219-24. doi:10.1016/J.Actatropica. 2009.07.030.

17. Smulian AG, Sullivan DW, Linke MJ, Halsey NA, Quinn TC, MacPhail AP, et al. Geographic variation in the humoral response to Pneumocystis Carinii. J Infect Dis. 1993;167(5):1243-7.

18. Lucas SB, Peacock CS, Hounnou A, Brattegaard K, Koffi K, Honde M, et al. Disease in children infected with HIV in Abidjan, Cote D'ivoire. BMJ. 1996; 312(7027):335-8.

19. Ruffini DD, Madhi SA. The high burden of Pneumocystis Carinii pneumonia in African HIV-1-infected children hospitalized for severe pneumonia. Aids. 2002;16(1):105-12. Epub 2001/12/13.

20. Madhi SA, Petersen K, Madhi A, Khoosal M, Klugman KP. Increased disease burden and antibiotic resistance of bacteria causing severe communityacquired lower respiratory tract infections in human immunodeficiency virus type 1-infected children. Clin Infect Dis. 2000;31(1):170-6. doi:10.1086/ 313925. Epub 2000/07/29.

21. Fisk DT, Meshnick S, Kazanjian PH. Pneumocystis Carinii pneumonia in patients in the developing world who have acquired immunodeficiency syndrome. Clin Infect Dis. 2003;36(1):70-8. doi:10.1086/344951.

22. Wasserman S, Engel ME, Mendelson M. Burden of Pneumocystis pneumonia in HIV-infected adults in Sub-Saharan Africa: protocol for a systematic review. Syst Rev. 2013;2:112. doi:10.1186/2046-4053-2-112. Epub 2013/12/18.

23. World Health Organization. Who case definitions of HIV for surveillance and revised clinical staging and immunological classification of HIV-related disease in adults and children. Geneva: World Health Organization; 2007.

24. Higgins JP, Thompson SG. Quantifying heterogeneity in a meta-analysis. Stat Med. 2002;21(11):1539-58. doi:10.1002/Sim.1186. Epub 2002/07/12.

25. Batungwanayo J, Taelman H, Lucas S, Bogaerts J, Alard D, Kagame A, et al. Pulmonary disease associated with the human immunodeficiency virus in Kigali, Rwanda. A fiberoptic bronchoscopic study of 111 cases of undetermined etiology. Am J Respir Crit Care Med. 1994;149(6):1591-6. doi:10.1164/Ajrccm.149.6.8004318. Epub 1994/06/01.

26. Kamanfu G, Mlika-Cabanne N, Girard PM, Nimubona S, Mpfizi B, Cishako A et al. Pulmonary complications of human immunodeficiency virus infection in Bujumbura, Burundi. Am Rev Respir Dis. 1993;147(3):658-63. doi:10.1164/ Ajrccm/147.3.658. Epub 1993/03/01.

27. Abouya YL, Beaumel A, Lucas S, Dago-Akribi A, Coulibaly G, N'Dhatz M, et al Pneumocystis Carinii Pneumonia. An uncommon cause of death in African patients with acquired immunodeficiency syndrome. Am Rev Respir Dis. 1992;145(3):617-20. doi:10.1164/Ajrccm/145.3.617. Epub 1992/03/01.

28. Buchacz K, Baker RK, Palella FJ, Jr., Chmiel JS, Lichtenstein KA, Novak RM, et al. Aids-defining opportunistic illnesses in us patients, 1994-2007: A cohort study. Aids. 2010;24(10):1549-59. doi:10.1097/Qad.0b013e32833a3967. Epub 2010/05/27.

29. Antiretroviral Therapy Cohort Collaboration, Mocroft A, Sterne JA, Egger M, May M, Grabar S, et al. Variable impact on mortality of aids-defining events diagnosed during combination antiretroviral therapy: not all aids-defining conditions are created equal. Clin Infect Dis. 2009;48(8):1138-51. doi:10.1086/597468.

30. de Armas Rodriguez Y, Wissmann G, Muller AL, Pederiva MA, Brum MC, Brackmann RL, et al. Pneumocystis Jirovecii pneumonia in developing countries. Parasite (Paris, France). 2011;18(3):219-28. Epub 2011/09/07.

31. Roux P, Lavrard I, Poirot JL, Chouaid C, Denis M, Olivier JL, et al. Usefulness of PCR for detection of Pneumocystis Carinii DNA. J Clin Microbiol. 1994; 32(9):2324-6. Epub 1994/09/01.

32. Harris JR, Marston BJ, Sangrujee N, DuPlessis D, Park B, et al. Costeffectiveness analysis of diagnostic options for Pneumocystis pneumonia (PCP). Plos One. 2011;6(8):E23158. doi:10.1371/Journal.Pone. 0023158.

33. Wood BR, Komarow L, Zolopa AR, Finkelman MA, Powderly WG, Sax PE. Test performance of blood beta-glucan for Pneumocystis Jirovecii pneumonia in 
patients with aids and respiratory symptoms. Aids (London, England). 2013;27(6):967-72. doi:10.1097/Qad.0b013e32835cb646.

34. Straetemans M, Glaziou P, Bierrenbach AL, Sismanidis C, van der Werf MJ. Assessing tuberculosis case fatality ratio: a meta-analysis. Plos One. 2011;6(6): E20755. doi:10.1371/Journal.Pone.0020755.

35. Scott JAG, Hall AJ, Muyodi C, Lowe B, Ross M, Chohan B, et al. Aetiology, outcome, and risk factors for mortality among adults with acute pneumonia in Kenya. Lancet. 2000;355(9211):1225-30. doi:10.1016/50140-6736(00)02089-4.

36. Walzer PD, Evans HE, Copas AJ, Edwards SG, Grant AD, Miller RF. Early predictors of mortality from Pneumocystis Jirovecii pneumonia in HIV-infected patients: 1985-2006. Clin Infect Dis. 2008;46(4):625-33. doi:10.1086/526778. Epub 2008/01/15.

Submit your next manuscript to BioMed Central and we will help you at every step:

- We accept pre-submission inquiries

- Our selector tool helps you to find the most relevant journal

- We provide round the clock customer support

- Convenient online submission

- Thorough peer review

- Inclusion in PubMed and all major indexing services

- Maximum visibility for your research

Submit your manuscript at www.biomedcentral.com/submit
Biomed Central 